International Journal of Pure and Applied Mathematics

Volume 112 No. $3 \quad 2017,481-488$

ISSN: 1311-8080 (printed version); ISSN: 1314-3395 (on-line version)

url: http://www.ijpam.eu

doi: 10.12732 /ijpam.v112i3.3

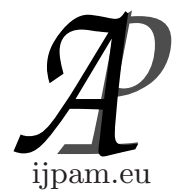

\title{
NOTES ON ZYGMUND FUNCTIONS
}

\author{
H. Akhadkulov ${ }^{1}$, M.S. Noorani ${ }^{2}$, A.B. Saaban ${ }^{3}$, S. Akhatkulov ${ }^{4}$ \\ ${ }^{1,3}$ School of Quantitative Sciences \\ University Utara Malaysia \\ CAS 06010, UUM Sintok, Kedah Darul Aman, MALAYSIA \\ ${ }^{2,4}$ School of Mathematical Sciences \\ University Kebangsaan \\ 43600 UKM Bangi, Selangor DE, MALAYSIA
}

\begin{abstract}
In this paper we study a class of continuous functions satisfying a certain Zygmund condition dependent on a parameter $\gamma>0$. It shown that the modulus of continuity of such functions is $\mathcal{O}\left(\delta\left(\log \frac{1}{\delta}\right)^{1-\gamma}\right)$ if $\gamma \in(0,1)$ and $\mathcal{O}\left(\delta\left(\log \log \frac{1}{\delta}\right)\right)$ if $\gamma=1$. Moreover, these functions are differentiable if $\gamma>1$. These results extend the results in literatures [4], [5].
\end{abstract}

AMS Subject Classification: 26A15, 42A55, 46E35

Key Words: Zygmund functions, modulus of continuity, differentiability

\section{Introduction}

One of most studied class of functions in harmonic analysis is the Zygmund class. This class is defined by "smooth" functions. Let $I$ be an open, finite interval on the line. Consider a function $\mathcal{K}: I \rightarrow \mathbb{R}$. Denote by $\Delta^{2} \mathcal{K}\left(x_{0}, \tau\right)$ the second symmetric difference of $\mathcal{K}$ that is,

$$
\Delta^{2} \mathcal{K}\left(x_{0}, \tau\right)=\mathcal{K}\left(x_{0}+\tau\right)+\mathcal{K}\left(x_{0}-\tau\right)-2 \mathcal{K}\left(x_{0}\right),
$$

where $x_{0} \in I$ and $\tau \in\left[0, \frac{|I|}{2}\right]$.

\footnotetext{
Received: $\quad$ August 30, 2016

Revised: $\quad$ November 21, 2016

Published: $\quad$ February 7, 2017

(c) 2017 Academic Publications, Ltd. url: www.acadpubl.eu

${ }^{\S}$ Correspondence author
} 
We shall say that $\mathcal{K}$ is "smooth" at $x_{0} \in \mathbb{R}$ if it satisfies

$$
\Delta^{2} \mathcal{K}\left(x_{0}, \tau\right)=o(\tau) \text { as } \tau \rightarrow 0
$$

where $o$ is a small o notation. It follows immediately that if $\mathcal{K}^{\prime}$ exists and is finite then $\mathcal{K}$ is "smooth" at $x_{0}$. The converse is obviously false, but if $\mathcal{K}$ is smooth at $x_{0}$ and if a one-sided derivative $\mathcal{K}$ at $x_{0}$ exists the derivative on the other side also exists and both are equal. If $\mathcal{K}$ is "smooth" at every point of $I$, we say that $\mathcal{K}$ is a "smooth" function on $I$. If $\mathcal{K}$ is continuous and satisfies (1) uniformly in $x_{0}$ i.e.,

$$
\left\|\Delta^{2} \mathcal{K}(\cdot, \tau)\right\|_{\infty}=o(\tau) \text { as } \tau \rightarrow 0
$$

we shall say that $\mathcal{K}$ is uniformly "smooth", where $\|\cdot\|_{\infty}$ is the supremum norm on $I$. The class of such functions is denoted by $\lambda_{*}$. Similarly, denote by $\Lambda_{*}$ the class of continuous functions $\mathcal{K}$ which satisfy the following relation uniformly in $x_{0}$

$$
\left\|\Delta^{2} \mathcal{K}(\cdot, \tau)\right\|_{\infty}=O(\tau) \text { as } \tau \rightarrow 0 .
$$

where $\mathcal{O}$ is a big $\mathrm{O}$ notation. The conditions (2) and (3) are called small Zygmund and Zygmund conditions, respectively. Furthermore, denote by $\Lambda_{\alpha}$ the class of functions satisfying the $\alpha$-Hölder condition of order $\alpha$ in $I$. Next we compare Zygmund classes with well known classes of analysis in order to be well understood for the reader the importance of these classes. It is easy to verify that if a function $g$ is continuously differentiable then it belongs to both of these classes $\lambda_{*}, \Lambda_{*}$ and if $g$ is Lipschitz then it belongs only to the class $\Lambda_{*}$. The reverse is not true. For example, the function $g(x)=x \log (x)+A x, x>0$ satisfies Zygmund condition but it is not Lipschitz. It is, however, $\alpha$-Hölder for each $\alpha<1$. If $g$ has bounded variation then it also does not necessarily belong to the class $\Lambda_{*}$ and vice versa. For example, the function $g(x)=\sqrt{x}$ has bounded variation but does not belong to the class $\Lambda_{*}$, and on the other hand the function $g(x)=x^{2} \sin \left(1 / x^{2}\right)$ has unbounded variation but it belongs to the class $\Lambda_{*}$. The following theorem shows that the modulus of continuity of the functions of classes $\lambda_{*}, \Lambda_{*}$ are "considerable".

Theorem 1.1. ([5], p.44) $\mathcal{K}$ be defined in a finite interval $I$. If $\mathcal{K} \in \Lambda_{*}$ then

$$
\omega(\delta ; \mathcal{K})=\mathcal{O}\left(\delta \log \frac{1}{\delta}\right)
$$

and in particular $\mathcal{K} \in \Lambda_{\alpha}$ for every $\alpha \in(0,1)$. If $\mathcal{K} \in \lambda_{*}$ then

$$
\omega(\delta ; \mathcal{K})=o\left(\delta \log \frac{1}{\delta}\right)
$$


where $\omega(\cdot ; \mathcal{K})$ is the modulus of continuity of $\mathcal{K}$.

The purpose of this work is to generalize the above theorem for a subclass of $\lambda_{*}$ which is defined as follows. Consider the function $\mathcal{Z}_{\gamma}:[0,1) \rightarrow(0,+\infty)$, given

$$
\mathcal{Z}_{\gamma}(x)=\frac{1}{\left(\log \frac{1}{x}\right)^{\gamma}}, \quad x \in(0,1)
$$

and $\mathcal{Z}_{\gamma}(0)=0$, where $\gamma>0$. Denote by $\widehat{\lambda}_{*}(\gamma)$ the class of continuous functions $\mathcal{K}: I \rightarrow \mathbb{R}$ satisfying

$$
\left\|\Delta^{2} \mathcal{K}(\cdot, \tau)\right\|_{\infty} \leq C \tau \mathcal{Z}_{\gamma}(\tau)
$$

for some constant $C:=C(\mathcal{K})>0$. Note that $\widehat{\lambda}_{*}(\gamma) \subset \lambda_{*}$ for all $\gamma>0$, since $\mathcal{Z}_{\gamma}(\tau) \rightarrow 0$ as $\tau \rightarrow 0$ and this class was investigated by Weiss and Zygmund [4]. They proved the following theorem.

Theorem 1.2. [4] Let $\mathcal{K}: \mathbb{R} \rightarrow \mathbb{R}$ be $2 \pi$-periodic and satisfies (4) for some $\gamma>\frac{1}{2}$. Then $\mathcal{K}$ is absolute continuous and $\mathcal{K} \in L_{p}[0,2 \pi]$ for every $p>1$.

More direct and general proof of this theorem can be found in [2]. In this theorem the assumption $\gamma>\frac{1}{2}$ is crucial. The theorem is false for the case of $\gamma \in\left(0, \frac{1}{2}\right]$. Below we shall provide an example satisfying (4) for some $\gamma \in\left(0, \frac{1}{2}\right]$ but almost nowhere differentiable.

In this work we study the class of functions $\widehat{\lambda}_{*}(\gamma)$ for different $\gamma$ 's. More precisely, we show that the modulus of continuity of the functions of $\widehat{\lambda}_{*}(\gamma)$ for $\gamma \in(0,1)$ is $\mathcal{O}\left(\delta\left(\log \frac{1}{\delta}\right)^{1-\gamma}\right)$ and for $\gamma=1$ is $\mathcal{O}\left(\delta\left(\log \log \frac{1}{\delta}\right)\right)$. This result extends Theorem 1.1. Moreover, we prove the differentiability of the functions of $\widehat{\lambda}_{*}(\gamma)$ for $\gamma>1$. This result generalizes the Weiss-Zygmund theorem for $\gamma>1$.

\section{Main Results}

In this section we prove our main results. For this we define the function $\mathcal{P}_{\gamma}:(0,1) \rightarrow \mathbb{R}$ as

$$
\mathcal{P}_{\gamma}(x)=\sum_{n=1}^{\infty} \mathcal{Z}_{\gamma}\left(x 2^{-n}\right) \text { where } x \in(0,1) \text { and } \gamma>1
$$

which will be needed in the proof of main theorems. It is clear that $\mathcal{P}_{\gamma}$ is continuous and $\lim _{x \rightarrow 0} \mathcal{P}_{\gamma}(x)=0$. The first main result is the following. 
Theorem 2.1. Let $\mathcal{K}: I \rightarrow \mathbb{R}$ be continuous and satisfies the inequality (4) on I. Then

$$
\omega(\delta, \mathcal{K}) \leq C \begin{cases}\delta\left(\log \frac{1}{\delta}\right)^{1-\gamma} & \text { if } \gamma \in(0,1) \\ \delta\left(\log \log \frac{1}{\delta}\right) & \text { if } \gamma=1 \\ \delta & \text { if } \gamma>1 ;\end{cases}
$$

where $\omega(\cdot, \mathcal{K})$ is the modulus of continuity of $\mathcal{K}$.

Proof. The proof follows closely that of [5] (p. 44). Let us consider the function $\mathcal{D}_{\tau} \mathcal{K}(x)=\mathcal{K}(x+\tau)-\mathcal{K}(x)$. Take any $x \in I$ we fix. The inequality (4) implies

$$
\left|\mathcal{D}_{\tau} \mathcal{K}(x)-2 \mathcal{D}_{\tau 2^{-1}} \mathcal{K}(x)\right| \leq C \tau \mathcal{Z}_{\gamma}(\tau)
$$

for small enough $\tau \in(0, \zeta]$. Replacing here $\tau$ successively by $\tau 2^{-1}, \tau 2^{-2}, \ldots$ ,$\tau 2^{-(n-1)}$ and in each step multiplying $2,2^{2}, \ldots, 2^{n-1}$ we obtain $\left|\mathcal{D}_{\tau} \mathcal{K}(x)-2 \mathcal{D}_{\tau 2^{-1}} \mathcal{K}(x)\right| \leq C \tau \mathcal{Z}_{\gamma}\left(\tau 2^{-1}\right)$,

$$
\begin{gathered}
\left|2 \mathcal{D}_{\tau 2^{-1}} \mathcal{K}(x)-2^{2} \mathcal{D}_{\tau 2^{-2}} \mathcal{K}(x)\right| \leq C \tau \mathcal{Z}_{\gamma}\left(\tau 2^{-2}\right) \\
\ddots \\
\left|2^{n-1} \mathcal{D}_{\tau 2^{-(n-1)}} \mathcal{K}(x)-2^{n} \mathcal{D}_{\tau 2^{-n}} \mathcal{K}(x)\right| \leq C \tau \mathcal{Z}_{\gamma}\left(\tau 2^{-n}\right) .
\end{gathered}
$$

Below $n$ and $\tau$ will be chosen. By termwise addition above inequalities we get

$$
\left|\mathcal{D}_{\tau} \mathcal{K}(x)-2^{n} \mathcal{D}_{\tau 2^{-n}} \mathcal{K}(x)\right| \leq C \tau \sum_{k=1}^{n} \mathcal{Z}_{\gamma}\left(\tau 2^{-k}\right)
$$

Suppose $\delta>0$ be sufficiently small. Let $0<\delta \leq \frac{1}{2} \zeta$ and $n$ be a natural such that $\frac{\zeta}{2} \leq 2^{n} \delta<\zeta$. This implies $n<\log _{2} \frac{\zeta}{\delta}$. Make substitution $\tau=2^{n} \delta$ in (6) we get

$$
\left|\mathcal{D}_{\delta} \mathcal{K}(x)\right| \leq \frac{2 \max _{x \in I}|\mathcal{K}(x)| \delta}{2^{n} \delta}+C \delta \sum_{k=1}^{n} \mathcal{Z}_{\gamma}\left(\delta 2^{n-k}\right) .
$$

Easily can be seen

$$
\delta \sum_{k=1}^{n} \mathcal{Z}_{\gamma}\left(\delta 2^{n-k}\right)=\delta \sum_{k=1}^{n} \frac{2^{-(n-k)} \mathcal{Z}_{\gamma}\left(\delta 2^{n-k}\right)}{2^{-(n-k)}} \leq C \int_{2^{-n}}^{1} \frac{\delta \mathcal{Z}_{\gamma}\left(\frac{\delta}{x}\right)}{x} d x
$$


An easy computation shows that

$$
\int_{2^{-n}}^{1} \frac{\delta \mathcal{Z}_{\gamma}\left(\frac{\delta}{x}\right)}{x} d x \leq C \begin{cases}\delta\left(\log \frac{1}{\delta}\right)^{1-\gamma} & \text { if } \gamma \in(0,1) \\ \delta\left(\log \log \frac{1}{\delta}\right) & \text { if } \gamma=1\end{cases}
$$

On the other hand

$$
\sum_{k=1}^{n} \mathcal{Z}_{\gamma}\left(\delta 2^{n-k}\right) \leq \mathcal{P}_{\gamma}(\delta)
$$

for $\delta \in\left[0, \frac{\zeta}{2}\right]$. Moreover $\mathcal{P}_{\gamma}$ is bounded since it is continuous on $\left[0, \frac{\zeta}{2}\right]$. This and the relations (7)-(10) imply the proof of Theorem 2.1 .

Now we provide a function satisfying (4) but almost nowhere differentiable.

Example. Consider a class of Weierstrass functions.

$$
\mathcal{W}_{\beta}(x)=\sum_{n=1}^{\infty} \theta_{n} b^{-\beta n} \cos \left(b^{n} x\right) \quad \text { where } \quad b>1 \text { and } \lim _{n \rightarrow \infty} \theta_{n}=0 .
$$

Weierstrass showed that for a small enough $\beta>0$ the function $\mathcal{W}_{\beta}$ is nowhere differentiable. The extension to $\beta \leq 1$ was first proved by Hardy. For $\beta>1$ the function $\mathcal{W}_{\beta}^{\prime}$ exists and continuous. If the sum of squares of the sequence $\theta_{n}$ is divergence then $\mathcal{W}_{1}$ is differentiable in a set of measure zero only. Thus, making $b:=2$ and instead of $\theta_{n}$ taking the sequence $n^{-\gamma}$ where $\gamma \in\left(0, \frac{1}{2}\right]$ we show that the function $\mathcal{W}_{1}$ satisfies (4). Indeed,

$$
\begin{gathered}
\mathcal{W}_{1}(x+\tau)+\mathcal{W}_{1}(x-\tau)-2 \mathcal{W}_{1}(x)=-4 \sum_{n=1}^{\infty} \frac{\cos \left(2^{n} x\right)}{2^{n} n^{\gamma}} \sin ^{2}\left(\frac{2^{n} \tau}{2}\right)= \\
-4 \sum_{n=1}^{N} \frac{\cos \left(2^{n} x\right)}{2^{n} n^{\gamma}} \sin ^{2}\left(\frac{2^{n} \tau}{2}\right)+(-4) \sum_{n=N+1}^{\infty} \frac{\cos \left(2^{n} x\right)}{2^{n} n^{\gamma}} \sin ^{2}\left(\frac{2^{n} \tau}{2}\right):=I_{1}+I_{2}
\end{gathered}
$$

where $N:=N(\tau)$ is the largest integer satisfying $2^{N} \tau \leq 1$, so that $2^{N+1} \tau>1$. Next we estimate $I_{1}, I_{2}$ separately. It is obvious

$$
\left|I_{1}\right| \leq 4 \sum_{n=1}^{N} \frac{1}{2^{n} n^{\gamma}}\left(\frac{2^{n} \tau}{2}\right)^{2}=\tau^{2} \sum_{n=1}^{N} \frac{2^{n}}{n^{\gamma}}=\tau^{2} 2^{N} \sum_{n=1}^{N} \frac{1}{2^{N-n} n^{\gamma}} \leq \tau \sum_{n=1}^{N} \frac{1}{2^{N-n} n^{\gamma}} .
$$

On the other hand

$$
\sum_{n=1}^{N} \frac{1}{2^{N-n} n^{\gamma}}=\sum_{n=1}^{\left[\frac{N}{2}\right]} \frac{1}{2^{N-n} n^{\gamma}}+\sum_{n=\left[\frac{N}{2}\right]+1}^{N} \frac{1}{2^{N-n} n^{\gamma}} \leq
$$




$$
\frac{2}{2^{\left[\frac{N}{2}\right]}}+\frac{1}{\left(\left[\frac{N}{2}\right]+1\right)^{\gamma}} \leq C\left(\sqrt{\tau}+\frac{1}{\left(\log \frac{1}{\tau}\right)^{\gamma}}\right) \leq C \mathcal{Z}_{\gamma}(\tau) .
$$

Thus $I_{1} \leq C \tau \mathcal{Z}_{\gamma}(\tau)$. It is easy to see

$$
\left|I_{2}\right| \leq 4 \sum_{n=N+1}^{\infty} \frac{1}{2^{n} n^{\gamma}} \leq \frac{4}{2^{N}(N+1)^{\gamma}} \leq C \tau \mathcal{Z}_{\gamma}(\tau)
$$

Hence

$$
\left|\mathcal{W}_{1}(x+\tau)+\mathcal{W}_{1}(x-\tau)-2 \mathcal{W}_{1}(x)\right| \leq C \tau \mathcal{Z}_{\gamma}(\tau)
$$

The right hand side of this inequality does not depend on $x$. Therefore $\|\cdot\|_{\infty^{-}}$ norm of the left hand side bounded with $C \tau \mathcal{Z}_{\gamma}(\tau)$. Obviously the total variation of this function is unbounded. This is the example which we have mentioned in the first section.

We now show, as a second main result that the function $\mathcal{K}$ is continuously differentiable and its modulus of continuity is $\mathcal{P}_{\gamma}$ for $\gamma>1$.

Theorem 2.2. Let $\mathcal{K}: I \rightarrow \mathbb{R}$ satisfies the inequality (4) for some $\gamma>1$. Then $\mathcal{K} \in C^{1}(I)$ and for any $\xi, \eta \in I$ there exists a constant $C>0$ such that

$$
\left|\mathcal{K}^{\prime}(\xi)-\mathcal{K}^{\prime}(\eta)\right| \leq C \cdot \mathcal{P}_{\gamma}(|\xi-\eta|)
$$

Proof. According to third item of Theorem 2.1, $\mathcal{K}$ is at least Lipschitz function in the case of $\gamma>1$. Hence $\mathcal{K}^{\prime}$ exists almost everywhere and $\mathcal{K}$ is the indefinite integral of $\mathcal{K}^{\prime}$. To prove this theorem we take any points $\xi, \eta \in I$ that are Lebesgue points of $\mathcal{K}^{\prime}$ and we obtain a uniform estimation for $\left|\mathcal{K}^{\prime}(\xi)-\mathcal{K}^{\prime}(\eta)\right|$. Hence we show $\mathcal{K}^{\prime}$ is uniformly continuous on its set of Lebesgue points, thus can be shown the continuity of $\mathcal{K}^{\prime}$. Let us consider the function $\mathcal{D}_{\tau} \mathcal{K}(x)=$ $\mathcal{K}(x+\tau)-\mathcal{K}(x)$ where $x, x+\tau \in I$. By inequality (4) we have

$$
\mathcal{D}_{\tau} \mathcal{K}(x)=\mathcal{D}_{\tau} \mathcal{K}(x-\tau)+\mathcal{O}\left(\tau \mathcal{Z}_{\gamma}(\tau)\right)
$$

for all $x \in I$ and for all $\tau \in[0,|I| / 2]$. Let us take any Lebesgue points $\xi, \eta \in I$ and fix them. We set $\tau:=|\xi-\eta|$. Replacing in (11) $x$ successively by $x_{n}=$ $\eta+\tau 2^{-n}$ and $\tau$ successively by $\tau 2^{-n}, n=1,2, \ldots$ we obtain

$$
\mathcal{D}_{\tau 2^{-n}} \mathcal{K}\left(x_{n}\right)=\mathcal{D}_{\tau 2^{-n}} \mathcal{K}(\eta)+\mathcal{O}\left(\tau 2^{-n} \mathcal{Z}_{\gamma}\left(\tau 2^{-n}\right)\right)
$$

It easily can be seen that for all $n \geq 1$ the following identity holds.

$$
\mathcal{D}_{\tau 2^{-n}} \mathcal{K}\left(x_{n}\right)-\mathcal{D}_{\tau 2^{-n}} \mathcal{K}(\eta)=\mathcal{D}_{\tau 2^{-n+1}} \mathcal{K}(\eta)-2 \mathcal{D}_{\tau 2^{-n}} \mathcal{K}(\eta)
$$


Thus

$$
\mathcal{D}_{\tau 2^{-n+1}} \mathcal{K}(\eta)=2 \mathcal{D}_{\tau 2^{-n}} \mathcal{K}(\eta)+\mathcal{O}\left(\tau 2^{-n} \mathcal{Z}_{\gamma}\left(\tau 2^{-n}\right)\right)
$$

This implies

$$
2^{-n+1} \mathcal{D}_{\tau 2^{-n+1}} \mathcal{K}(\eta)=2^{-n} \mathcal{D}_{\tau 2^{-n}} \mathcal{K}(\eta)+\mathcal{O}\left(\tau \mathcal{Z}_{\gamma}\left(\tau 2^{-n}\right)\right) .
$$

By termwise addition from $n=1$ to $N$ and divide $\tau$ we obtain

$$
\frac{\mathcal{D}_{\tau} \mathcal{K}(\eta)}{\tau}=\frac{2^{N}}{\tau} \mathcal{D}_{\tau 2^{-N}} \mathcal{K}(\eta)+\mathcal{O}\left(\sum_{n=1}^{N} \mathcal{Z}_{\gamma}\left(\tau 2^{-n}\right)\right) .
$$

Since the point $\eta$ is the Lebesgue point of $\mathcal{K}^{\prime}$ and $\gamma>1$ taking limit as $N \rightarrow \infty$ in $(12)$ we get

$$
\lim _{N \rightarrow \infty} \frac{2^{N}}{\tau} \mathcal{D}_{\tau 2^{-N}} \mathcal{K}(\eta)=\mathcal{K}^{\prime}(\eta)
$$

and

$$
\frac{\mathcal{D}_{\tau} \mathcal{K}(\eta)}{\tau}=\mathcal{K}^{\prime}(\eta)+\mathcal{O}\left(\mathcal{P}_{\gamma}(\tau)\right)
$$

Similarly as above, replacing in (11) $x$ successively by $x_{n}=\xi-\tau 2^{-n}$ and $\tau$ successively by $\tau 2^{-n}, n=1,2, \ldots$ we obtain

$$
\mathcal{D}_{\tau 2^{-n}} \mathcal{K}\left(x_{n}\right)=\mathcal{D}_{\tau 2^{-n}} \mathcal{K}\left(x_{n-1}\right)+\mathcal{O}\left(\tau 2^{-n} \mathcal{Z}_{\gamma}\left(\tau 2^{-n}\right)\right)
$$

Here the following identity can be also easily checked for all $n \geq 1$.

$$
\mathcal{D}_{\tau 2^{-n}} \mathcal{K}\left(x_{n}\right)-\mathcal{D}_{\tau 2^{-n}} \mathcal{K}\left(x_{n-1}\right)=-\left(\mathcal{D}_{\tau 2^{-n+1}} \mathcal{K}\left(x_{n-1}\right)-2 \mathcal{D}_{\tau 2^{-n}} \mathcal{K}\left(x_{n}\right)\right) .
$$

This implies

$$
2^{-n+1} \mathcal{D}_{\tau 2^{-n+1}} \mathcal{K}\left(x_{n-1}\right)=2^{-n} \mathcal{D}_{\tau 2^{-n}} \mathcal{K}\left(x_{n}\right)+\mathcal{O}\left(\tau \mathcal{Z}_{\gamma}\left(\tau 2^{-n}\right)\right) .
$$

The same manner as above we get

$$
\frac{\mathcal{D}_{\tau} \mathcal{K}(\xi-\tau)}{\tau}=\mathcal{K}^{\prime}(\xi)+\mathcal{O}\left(\mathcal{P}_{\gamma}(\tau)\right)
$$

So far as the right hand sides of (13) and (14) are same. Therefore we have

$$
\left|\mathcal{K}^{\prime}(\xi)-\mathcal{K}^{\prime}(\eta)\right| \leq C \cdot \mathcal{P}_{\gamma}(|\xi-\eta|)
$$

This proves uniform continuity of $\mathcal{K}^{\prime}$ on the set of Lebesgue points, thus $\mathcal{K}^{\prime}$ coincides almost everywhere on $I$ with a some continuous function $\mathcal{U}: I \rightarrow \mathbb{R}$. It is clear $\int_{a}^{x} \mathcal{U}(t) d t$ is a $C^{1}(I)$ function and the function $\mathcal{L}(x):=\int_{a}^{x} \mathcal{U}(t) d t-\mathcal{K}(x)$ is absolute continuous. However, $\mathcal{L}^{\prime}(x)=0$ almost everywhere, hence $\mathcal{L}(x) \equiv$ const. Theorem 2.2 is therefore completely proved. 
Remark 2.3. Note that

- Theorem 2.2 extends the third item of Theorem 2.1 for $\gamma>1$.

- Theorem 2.2 generalizes The Weiss-Zygmund theorem (Theorem 1.2) for $\gamma>1$.

- The Zygmund classes are not only important classes in harmonic analysis, but they have deserved applications in the theory circle maps. Sullivan and $\mathrm{Hu}[3]$ who first applied the Zygmund classes to the theory of circle maps.

- Recently, the authors have applied the class $\widehat{\lambda}_{*}(\gamma)$ to the theory of renormalizations (see [1]).

\section{Acknowledgments}

The authors are very grateful to the Ministry of Higher Education of Malaysia for providing us with the Fundamental Research Grant Scheme (FRGS), S/O number 13558 to enable us pursue this research.

\section{References}

[1] H. Akhadkulov, M.S. Noorani, S. Akhatkulov, Renormalization of circle diffeomorphisms with a break-type singularity. Preprint, (2016), 26-pages, Avaliable on: http://arxiv.org/pdf/1510.03202v2.pdf

[2] F. John, L. Nirenberg, On functions of bounded mean oscillation, Comm. Pure Appl. Math., 14, (1961), 415-426.

[3] J. Hu, D. Sullivan, Topological conjugacy of circle diffeomorphisms, Ergodic Theory Dynam. Systems, 17, No. 1 (1997), 173-186.

[4] M. Weiss, A. Zygmund, A note on smooth functions, Indag. Math., 62 (1959), 52-58.

[5] A. Zygmund, Trigonometric Series, Third Edition, Volumes I and II, Cambridge University Press, London, 2002. 